\title{
AÇÕES DE ENFERMAGEM PODEM PREVENIR DEISCÊNCIA EM FERIDA OPERATÓRIA?
}

\author{
Can nursing actions prevent surgical wound dehiscence?
}

\author{
¿Pueden las acciones de enfermería prevenir la dehiscencia de la herida operatoria? \\ Eduardo Tavares Gomes ${ }^{1 *}$ (1) , Vanessa de Brito Poveda ${ }^{2}$ (D) Vilanice Alves de Araújo Püschel ${ }^{3}$ (1)
}

RESUMO: Objetivo: Identificar na literatura científica intervenções de enfermagem úteis para a prevenção de Deiscências em Feridas Cirúrgicas. Método: Trata-se de uma revisão integrativa da literatura, norteada pela pergunta: Há ações de enfermagem que possam contribuir para prevenção de deiscência em ferida operatória? Realizou-se a busca no período de março de 2019, incluindo artigos publicados a partir do ano de 1990 até 2018. Para a seleção dos artigos foram utilizadas as seguintes bases de dados e/ ou portais: National Library of Medicine (PubMed); Web of Science; Scopus Info Site (Scopus); Literatura Latino-Americana e do Caribe em Ciência da Saúde (LILACS) e Cumulative Index to Nursing and Allied Health Literature (CINHAL). Resultados: A busca resultou em 64 artigos, excluíram-se quatro por estarem duplicados e 40 por não atenderem aos critérios de inclusão (24 por serem sobre tratamento, 13 pela natureza do artigo, dois por não estarem disponíveis e um por ser em francês). Vinte artigos foram avaliados na íntegra e 14 foram excluídos por não responderem à questão norteadora desta revisão. Dessa maneira, ao fim desse processo de análise, foram selecionados seis artigos que responderam aos critérios de inclusão e constituíram a amostra final. Conclusão: Houve pouca produção da enfermagem sobre o tema. As principais ações de enfermagem para prevenção de deiscência em feridas cirúrgicas estão associadas à prevenção de infecções de sítio cirúrgico e à indicação e à utilização de terapia de cobertura a vácuo. Palavras-chave: Ferida cirúrgica. Deiscência da ferida operatória. Infecção da ferida cirúrgica. Enfermagem perioperatória. Assistência perioperatória.

ABSTRACT: Objective: To identify useful nursing interventions for preventing Surgical Wound Dehiscence in scientific literature. Method: This is an integrative literature review, guided by the question: are there nursing actions that can contribute to the prevention of surgical wound dehiscence? The search was carried out in March 2019, including articles published from 1990 to 2018. We used the following databases and/or portals to select the articles: National Library of Medicine (PubMed); Web of Science; Scopus Info Site (Scopus); Latin American and Caribbean Health Sciences Literature (LILACS), and Cumulative Index to Nursing and Allied Health Literature (CINHAL). Results: The search resulted in 64 articles. We excluded four of them for being duplicates, and another 40 that did not meet the inclusion criteria (24 were about treatment, 13 due to the nature of the article, two were not available, and one was in French). Twenty articles were fully evaluated, and 14 were excluded because they did not answer the guiding question of this review. Thus, at the end of this analysis process, we selected six articles that met the inclusion criteria and constituted the final sample. Conclusion: The nursing production on the subject was scarce. The main nursing actions for preventing surgical wound dehiscence are associated with the prevention of surgical site infections and the indication and use of negative pressure wound therapy.

Keywords: Surgical wound. Surgical wound dehiscence. Surgical wound infection. Perioperative nursing. Perioperative care.

RESUMEN: Objetivo: identificar en la literatura científica intervenciones de enfermería útiles para la prevención de la dehiscencia en heridas quirúrgicas. Método: Esta es una revisión de literatura integradora, guiada por la pregunta: ¿Existen acciones de enfermería que puedan contribuir a la prevención de la dehiscencia en las heridas quirúrgicas? La búsqueda se realizó en el período de marzo de 2019, incluidos los artículos publicados de 1990 a 2018 . Para la selección de artículos, se utilizaron las siguientes bases de datos y/o portales: National library of Medicine (PubMed); Web of Science; Scopus Info Site

'Enfermeiro assistencial da Unidade de Centro Cirúrgico do Hospital das Clínicas da Universidade Federal de Pernambuco (HC/UFPE). Doutorando em Ciências pelo Programa de Pós-graduação em Enfermagem em Saúde do Adulto da Escola de Enfermagem da Universidade de São Paulo (USP) - São Paulo (SP), Brasil.

2Professora associada do Departamento de Enfermagem Médico-Cirúrgica da Escola de Enfermagem da USP. Coordenadora do Programa de Pós-Graduação em Enfermagem na Saúde do Adulto (PROESA) da Escola de Enfermagem da USP - São Paulo (SP), Brasil.

${ }^{3}$ Professora Titular do Departamento de Enfermagem Médico-Cirúrgica da Escola de Enfermagem da USP - São Paulo (SP), Brasil.

*Autor correspondente: edutgs@hotmail.com

Recebido: 05/08/2019 - Aprovado: 30/01/2020

DOI: $10.5327 / Z 1414-4425202000020008$ 
(Scopus); Literatura latinoamericana y caribeña de ciencias de la salud (LILACS) y Cumulative Index to Nursing and Allied Health Literature (CINHAL). Resultados: La búsqueda resultó en 64 artículos, se excluyeron cuatro duplicados, 40 artículos por no cumplir con los criterios de inclusión (24 sobre el tratamiento, 13 por la naturaleza del artículo, dos por no estar disponibles y uno por estar en francés), y 20 los artículos fueron evaluados en su totalidad y 14 fueron excluidos porque no respondieron la pregunta guía de esta revisión. Por lo tanto, al final de este proceso de análisis, se seleccionaron seis artículos que cumplían los criterios de inclusión y constituían la muestra final. Conclusión: hubo poca producción de enfermería sobre el tema. Las principales acciones de enfermería para prevenir la dehiscencia en las heridas quirúrgicas están asociadas con la prevención de infecciones del sitio quirúrgico y la indicación y el uso de la terapia de cobertura con vacío.

Palabras clave: Herida quirúrgica. Dehiscencia de la herida operatoria. Infección de la herida quirúrgica. Enfermería perioperatoria. Atención perioperativa.

\section{INTRODUÇÃO}

A deiscência de feridas cirúrgicas (DFC) é uma complicação pós-operatória que prejudica a cicatrização das feridas e aumenta o tempo de internação e os custos hospitalares ${ }^{1-3}$, podendo ser definida como separação das margens de uma ferida fechada depois de um procedimento cirúrgico, que ocorre geralmente até 10 dias após a cirurgia, embora possa ocorrer até o $30^{\circ}$ dia ${ }^{4,5}$.

A incidência de DFC varia segundo os procedimentos cirúrgicos relacionados, tais como procedimentos abdominais (1,3 a 4,7\%), cesarianas (13,3\%), próteses de quadril (14,3\%) e cirurgias cardiotorácicas $(15,3 \%)^{1}$. As DFC estão associadas a mais morbidades e mortalidade e a mais fatores de risco ${ }^{6}$.

A cicatrização após uma deiscência é mais lenta, podendo o paciente necessitar de uma nova intervenção cirúrgica. De forma geral, pode-se afirmar que as DFC surgem em função de fatores técnicos (escolha do fio, incisão e técnica de sutura), de estresse mecânico (tosse, movimento abrupto ou vigoroso) e de problemas relacionados ao processo natural de cura ${ }^{7}$. Para cura da ferida operatória, conjugam-se fatores como oxigenação e perfusão no leito da ferida e aporte de nutrientes, que podem ser prejudicados pelo edema local, por infecção e por condições próprias da pele, como envelhecimento e alterações causadas por diabetes ${ }^{7,8}$.

A Infecção do Sítio Cirúrgico (ISC) está relacionada não apenas à deiscência, mas também ao surgimento de hérnias incisionais ${ }^{9}$. Além da relação com a ISC, a deiscência pode estar relacionada a causas não infecciosas, como coleta de hematoma ou seroma, fatores relacionados aos pacientes, como obesidade e diabetes e causas mecânicas, por exemplo resultado de traumas, crises de vômitos e tosse $\mathrm{e}^{2,4,10}$. Ademais, mesmo quando a causa não é infecciosa, após a deiscência, pode ocorrer infecção, o que dificulta ainda mais o processo de cura ${ }^{2,7,8}$.

Pesquisas com o intuito de desenvolver e validar um modelo de risco para DFC descreveram como fatores de risco independentes mais recorrentes: idade avançada, sexo biológico feminino, doença pulmonar crônica, edema, ascite, anemia, cirurgia de emergência, tipo de cirurgia, tosse no pós-operatório, tabagismo e infecção, além da relação direta entre a maior presença de fatores de risco e a maior chance de óbito ${ }^{4,11}$. Há evidências de mais incidência de deiscência quando, além da ferida operatória, há confecção de estomas ${ }^{12,13}$.

Considerando o impacto que as deiscências cirúrgicas causam no cuidado ao paciente no pós-operatório, a pesquisa na área deve buscar entender como prevenir esse evento, bem como desenvolver tecnologias que favoreçam a prevenção. Particularmente, à enfermagem cabe a responsabilidade de corroborar a construção desse conhecimento, visto que o enfermeiro atua diretamente no cuidado perioperatório e no cuidado da ferida no pós-operatório.

\section{OBJETIVO}

Identificar, na literatura científica nacional e internacional, intervenções de enfermagem úteis para a prevenção de DFC.

\section{MÉTODO}

Este estudo é uma revisão integrativa da literatura, um método de pesquisa que utiliza a prática baseada em evidências, sintetiza as pesquisas disponíveis de um assunto em questão e permite que o conhecimento científico direcione a prática ${ }^{14}$.

Para a realização deste estudo, foram utilizadas as etapas previstas da revisão integrativa: identificação do tema e elaboração da pergunta norteadora, busca na literatura com critérios de inclusão e de exclusão, definição das informações a serem extraídas dos estudos selecionados por meio de uma ficha bibliográfica previamente construída, coleta de dados, avaliação com análise crítica dos estudos incluídos na revisão, discussão dos resultados e apresentação da revisão integrativa ${ }^{14}$. 
A busca foi norteada pela pergunta: Há ações de enfermagem que possam contribuir para prevenção de deiscência em ferida operatória? Realizou-se a busca no mês de maço de 2019, que incluiu artigos publicados a partir do ano de 1990 até 2018, considerando que na década de 1990 houve grande incremento nas cirurgias digestivas abdominais, sobretudo nas bariátricas. Para a seleção dos artigos, foram utilizadas as seguintes bases de dados e/ ou portais: National Library of Medicine (PubMed), Web of Science; Scopus Info Site (Scopus), Literatura Latino-Americana e do Caribe em Ciência da Saúde (LILACS) e Cumulative Index to Nursing and Allied Health Literature (CINHAL).

Os critérios de inclusão foram: artigos publicados em português, inglês e espanhol, que relataram evidências na prevenção de deiscências em feridas operatórias. Os critérios de exclusão foram: estudos com outras complicações cirúrgicas (como granuloma e seroma) e artigos que não responderam ao objetivo desta revisão (artigos, editoriais e cartas ao editor que versem sobre tratamento da deiscência).

Para a busca, selecionaram-se descritores dos Descritores em Ciências da Saúde (DECS) e do Medical Subject Headings Section (MESH), sendo eles: surgical wound dehiscence, postoperative complications e nursing. Em virtude das características de acesso às bases de dados selecionadas, foram utilizadas estratégias combinadas de diferentes formas com o propósito de atingir uma busca ampla, tendo como eixos norteadores a questão do estudo e os critérios de inclusão previamente estabelecidos (Quadro 1).

Em todas as etapas de avaliação dos artigos resultantes da busca, participaram dois avaliadores com experiência em estudos de revisão, havendo um consenso para inclusão dos artigos. Foi realizada uma primeira avaliação dos artigos pelo título e pelo resumo. Para a coleta e análise de dados, utilizou-se um instrumento próprio, contendo os seguintes itens: título do artigo, título do periódico, autores, país, idioma, ano de publicação, tipo de estudo, objetivo, população de estudo, período de estudo, intervenção, método de avaliação, análise estatística, resultado e conclusão. Para a organização das referências encontradas o recurso utilizado foi o software EndNote ${ }^{\mathrm{TM}}$ versão web.

A avaliação dos estudos foi realizada de forma qualitativa. Para a avaliação metodológica dos estudos selecionados, utilizou-se o nível de evidência do Instituto Joanna Briggs para estudos de efetividade ${ }^{15}$. A síntese dos artigos é apresentada no tópico Resultados, em um quadro sinóptico contendo as seguintes características: autor/ano, periódico, título, desenho, resultado e nível de evidência (Quadro 2).

Quadro 1. Estratégias de busca de dados por base/portal.
Base de Dados
Estratégia de busca
Medline via PubMed
(“Surgical Wound Dehiscence"[Mesh] OR “Surgical Wound Dehiscence"[tw]) AND ("Nursing"[Mesh] OR "Nursing Care"[Mesh])
Scopus
("Surgical Wound Dehiscence" AND ("Postoperative Complications" OR "Postoperative Complication") AND (Nursing OR "Nursing Care")
Web of Science
TÓPICO: ("Surgical Wound Dehiscence" AND Nursing OR “Nursing Care")
LILACS mh: (“Deiscência da Ferida Operatória/NU”)
CINAHL
(MH “Surgical Wound Dehiscence/NU")

PubMed: National Library of Medicine; LILACS: Literatura Latino-Americana e do Caribe em Ciência da Saúde; CINAHL: Cumulative Index to Nursing and Allied Health Literature.

Quadro 2. Corpus de análise selecionado. Artigos selecionados como amostra do estudo, segundo autores, ano de publicação, periódico, título, desenho, resultado e nível de evidência.

\begin{tabular}{|l|c|c|c|c|c|}
\hline Autor (Ano) & Periódico & Título & Desenho & Nível de \\
Evidência
\end{tabular}


Quadro 2. Continuação.

\begin{tabular}{|c|c|c|c|c|c|}
\hline Autor (Ano) & Periódico & Título & Desenho & Resultado & $\begin{array}{l}\text { Nível de } \\
\text { Evidência }\end{array}$ \\
\hline $\begin{array}{l}\text { Scalise } \\
\text { et al., } 2016^{16}\end{array}$ & $\begin{array}{l}\text { International } \\
\text { Wound } \\
\text { Journal }\end{array}$ & $\begin{array}{l}\text { Improving wound healing } \\
\text { and preventing surgical site } \\
\text { complications of closed } \\
\text { surgical incisions: a possible } \\
\text { role of Incisional Negative } \\
\text { Pressure Wound Therapy: } \\
\text { a systematic review of the } \\
\text { literature }\end{array}$ & $\begin{array}{c}\text { Revisão } \\
\text { sistemática }\end{array}$ & $\begin{array}{c}\text { A literatura mostrou que a } \\
\text { terapia a vácuo diminuiu a } \\
\text { incidência de infecção, da } \\
\text { formação de sero-hematoma e } \\
\text { das taxas de reoperações. Menor } \\
\text { nível de evidência foi encontrado } \\
\text { para deiscências, sendo } \\
\text { inconclusiva a relação entre } \\
\text { terapia a vácuo e deiscências. }\end{array}$ & $\begin{array}{c}2 \mathrm{~b} \\
\text { (Revisão } \\
\text { sistemática } \\
\text { que incluiu até } \\
\text { estudos quase- } \\
\text {-experimentais) }\end{array}$ \\
\hline $\begin{array}{l}\text { Sandy- } \\
\text { Hodgetts } \\
\text { e Watts, } \\
2015^{17}\end{array}$ & $\begin{array}{l}\text { JBI Database } \\
\text { System Rev } \\
\text { Implement } \\
\text { Rep }\end{array}$ & $\begin{array}{l}\text { Effectiveness of negative } \\
\text { pressure wound therapy/closed } \\
\text { incision management in the } \\
\text { prevention of post-surgical wound } \\
\text { complications: a systematic } \\
\text { review and meta-analysis }\end{array}$ & $\begin{array}{c}\text { Revisão } \\
\text { sistemática }\end{array}$ & $\begin{array}{l}\text { Há evidências da relação entre } \\
\text { terapia a vácuo e redução de } \\
\text { infecção, mas não há evidências } \\
\text { diretas da mesma relação } \\
\text { com deiscências de feridas } \\
\text { operatórias. }\end{array}$ & $\begin{array}{c}\text { 1b } \\
\text { (Revisão } \\
\text { sistemática de } \\
\text { ensaios clínicos e } \\
\text { outros estudos) }\end{array}$ \\
\hline $\begin{array}{l}\text { Petito et al., } \\
2014^{18}\end{array}$ & $\begin{array}{l}\text { Journal } \\
\text { of Clinical } \\
\text { Nursing }\end{array}$ & $\begin{array}{l}\text { The influence of the initiation } \\
\text { of an exercise programme } \\
\text { on seroma formation and } \\
\text { dehiscence following breast } \\
\text { cancer surgery }\end{array}$ & $\begin{array}{l}\text { Ensaio } \\
\text { clínico } \\
\text { randomizado }\end{array}$ & $\begin{array}{l}\text { Mulheres que tiveram exercícios } \\
\text { de reabilitação precoce (antes da } \\
\text { retirada do dreno) não tiveram } \\
\text { desfechos diferentes. }\end{array}$ & $\begin{array}{l}\text { 1c } \\
\text { (Ensaio clínico } \\
\text { randomizado) }\end{array}$ \\
\hline $\begin{array}{l}\text { Blume et al., } \\
2010^{19}\end{array}$ & $\begin{array}{l}\text { International } \\
\text { Wound } \\
\text { Journal }\end{array}$ & $\begin{array}{l}\text { Retrospective evaluation } \\
\text { of clinical outcomes in } \\
\text { subjects with split-thickness } \\
\text { skin graft: comparing VAC }{ }^{\circledR} \\
\text { therapy and conventional } \\
\text { therapy in foot and ankle } \\
\text { reconstructive surgeries }\end{array}$ & $\begin{array}{l}\text { Descritivo } \\
\text { retrospectivo }\end{array}$ & $\begin{array}{l}\text { Curativo a vácuo teve associação } \\
\text { com menos complicações } \\
\text { (seroma, hematoma e infecção) } \\
\text { nos enxertos de pele. }\end{array}$ & $\begin{array}{l}\text { 3c } \\
\text { (Estudo de coorte } \\
\text { com grupo } \\
\text { controle) }\end{array}$ \\
\hline
\end{tabular}

\section{RESULTADOS}

A busca nas bases de dados selecionadas resultou em 64 artigos, dos quais excluíram-se quatro, por estarem duplicados, restando 60 artigos. Após leitura dos títulos e dos resumos, 40 artigos foram retirados por não atenderem aos critérios de inclusão (24 por serem sobre tratamento, 13 pela natureza do artigo, dois por não estarem disponíveis e um por ser em francês), restando 20 artigos. Destes, 14 artigos foram avaliados na íntegra e excluídos por não responderem à questão norteadora desta revisão. Dessa maneira, ao fim desse processo de análise, foram selecionados seis artigos que responderam aos critérios de inclusão e constituíram a amostra final. A Figura 1 descreve o processo de seleção e de inclusão dos artigos.

Apenas seis artigos compuseram o corpus de análise, sendo todos em inglês e publicados a partir de 2010, conforme apresentado no Quadro 2. Os artigos apresentam evidências da associação de deiscências a infecções, início precoce de exercícios de reabilitação antes da retirada dos drenos e uso de curativos a vácuo em feridas operatórias.

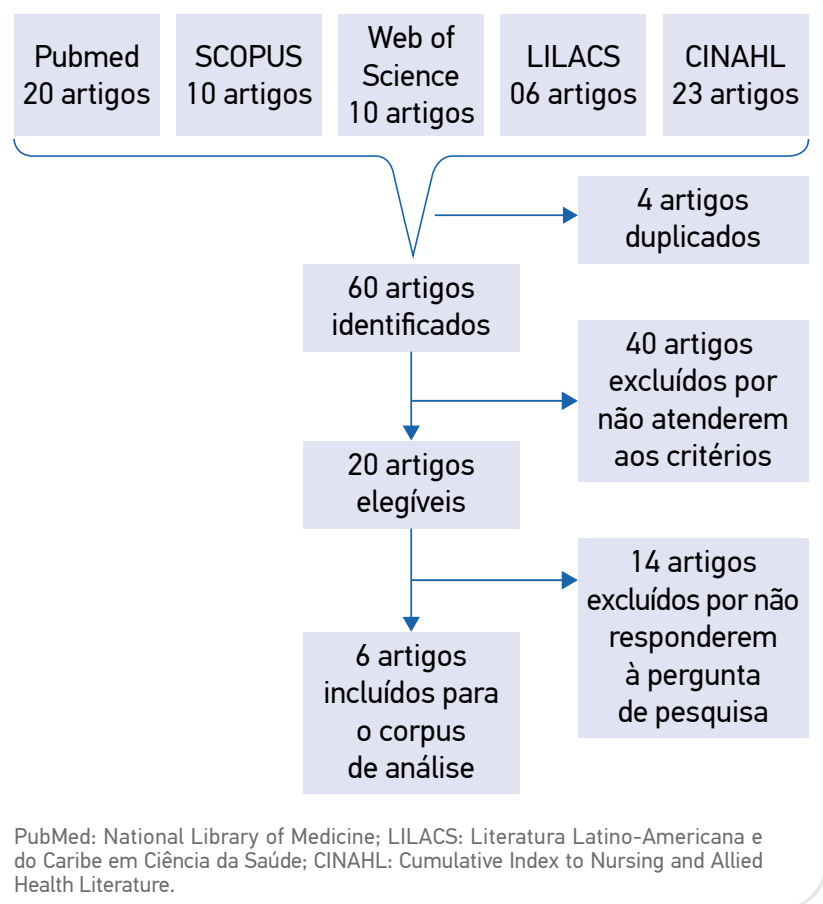

Figura 1. Fluxograma da seleção dos artigos que compuseram o corpus de análise. 


\section{DISCUSSÃO}

O cruzamento dos descritores evidenciou baixa produção científica da enfermagem acerca do tema. Além disso, os artigos que foram identificados não versavam diretamente sobre estratégias de prevenção de deiscência cirúrgica. Uma das revisões não foi incluída por não ter o método descrito de modo suficiente. Essa revisão não incluída apresentou que a detecção precoce de sinais de infecção e de seroma e a intervenção precoce são cruciais para a manutenção do estoma ${ }^{13}$. As outras revisões incluídas concluíram que a terapia a vácuo reduz a incidência de infecções, mas apontaram que as evidências de menor prevalência de deiscência associada ao uso desse método como medida profilática não eram suficientes ${ }^{2,16,17}$.

Para o fechamento de feridas cirúrgicas limpas, o método mais utilizado é a combinação de sutura com gaze para cobertura primária, contudo há disponível no mercado adesivos, grampos, hidrocoloides e outros dispositivos mais avançados ${ }^{2}$. Para feridas que não de pode aproximar as bordas para o fechamento tradicional ou para os casos de deiscência, a terapia de curativo a vácuo tem sido cada vez mais utilizada, apresentando bons resultados ${ }^{2}$.

No intuito de responder à questão norteadora, outras referências foram consultadas. A World Union of Wound Healing Societies (WUWHS) publicou, em 2018, um consenso a respeito da melhoria da prevenção e dos resultados das DFC, no qual apresenta os fatores de risco para DFC, classificando-os em fatores relacionados aos pacientes e fatores pré, intra e pós-operatórios ${ }^{20}$. Em análise acerca dos fatores de risco apresentados no documento, é possível afirmar que o enfermeiro pode, de forma direta, colaborar para a prevenção de deiscências, atuando na prevenção de hipotermia, na prevenção de complicações mecânicas, por meio da educação do paciente para tosse e para esforços no pós-operatório e da remoção da sutura no período adequado, e na prevenção de infecção da ferida operatória $^{20}$. Dos artigos encontrados, apenas um correlacionou ISC com DFC. Contudo, outras referências que avaliaram fatores de risco indicaram a ISC como fator significativo ${ }^{1,21-23}$.

$\mathrm{Na}$ fase pré-operatória, o enfermeiro deve considerar utilizar um instrumento para avaliar o risco de infecção e de deiscência da ferida operatória, bem como elaborar intervenções educativas para o paciente acerca do autocuidado no pós-operatório com feridas, drenos, tosses e esforços. De forma indireta, todas as ações pré-operatórias de prevenção de ISC impactam na prevenção de deiscências ${ }^{2023}$.

No intraoperatório, o enfermeiro, mesmo que não esteja no campo operatório, pode colaborar fiscalizando a adesão às técnicas assépticas, assegurando que não haja quebra dessa em nenhuma das etapas, por meio da observação e da cobrança desde o preparo correto da pele até a troca de luvas para o fechamento da ferida cirúrgica ${ }^{20}$.
O enfermeiro atua, ainda, na cobertura da ferida operatória. Omomentode cobertura da feridaéde extrema importância, podendo seroportuno paraidentificação de outraslesões de pele decorrentes do período intraoperatório ${ }^{24}$. Não houve estudos que pesquisassem essa temática, além do estudo com curativo a vácuo, contudo, os enfermeiros devem investigar se há formas mais eficazes de coberturas, avaliando novas tecnologias ou técnicas antigas e usualmente encontradas nos centros cirúrgicos, como uso de ataduras para aumentar a tensão na ferida do tórax, cintas para aumentar a tensão na parede abdominal e crioterapia. A pesquisa de enfermagem pode responder se essas técnicas e outras tecnologias podem diminuir a incidência de deiscências. O consenso da WUWHS orienta a cobertura das feridas operatórias por pelo menos 48 horas, ou menos, se houver necessidade de monitorização mais estrita de sinais e de sintomas precoces ${ }^{20}$. Os estudos de crioterapia estão mais relacionados ao alívio da dor do que à prevenção de infecção ou deiscência ${ }^{22}$.

Um dos artigos do corpus de análise evidenciou que exercícios precoces para pacientes ainda com drenos não tiveram resultados piores do que em pacientes que já os haviam retirado. Apesar de não trazer bom nível de evidência, para a enfermagem pode indicar que pesquisas devem ser realizadas sobre a deambulação precoce, de modo a considerá-la como fator de proteção no período intraoperatório ${ }^{18}$.

O atual guideline da Organização Mundial da Saúde para prevenção de ISC apresenta evidências da associação entre hipotermia e infecção e deiscência da ferida cirúrgica, além de diretrizes para intervenções para prevenção de hipotermia no período perioperatório ${ }^{25}$.

Após uma síntese de evidências, o consenso da WUWHS sugere que a terapia a vácuo antes do paciente deixar a sala de cirurgia seja instituída na prevenção de deiscência sempre que o paciente apresentar um fator de risco maior (índice de massa corporal $\geq 40 \mathrm{~kg}$ / $\mathrm{m}^{2}$, diabetes melito e procedimentos com alta incidência de deiscência) ou dois ou mais fatores de risco moderados ${ }^{20}$.

\section{CONCLUSÃO}

A busca na literatura revelou que a enfermagem pouca colabora na pesquisa acadêmica acerca do tema, visto que foram encontrados poucos artigos e com nível de evidência moderado.

Com base nos artigos e nas referências que corroboraram a discussão dos achados, pode-se afirmar, em resposta ao questionamento inicial desta revisão, que as principais ações de enfermagem na prevenção de DFC estão na prevenção de ISC e na indicação e na utilização de terapia de cobertura a vácuo. Devem ser pesquisadas outras formas de cobertura, bem como o uso de faixas, cintas e coberturas que aumentem a tensão na ferida operatória. 


\section{REFERÊNCIAS}

1. Sandy-Hodgetts K, Leslie G, Lewin G, Hendrie D, Carville, K. Surgical wound dehiscence in an Australian community nursing service: time and cost to healing. J Wound Care. 2016;25(7):377-83. http://doi. org/10.12968/jowc.2016.25.7.377

2. Stannard JP, Gabriel A, Lehner B. Use of negative pressure wound therapy over clean, closed surgical incisions. Int Wound J. 2012;9(s1):32-9. http://doi.org/10.1111/j.1742-481x.2012.01017.x

3. Shanmugam VK, Fernandez SJ, Evans KK, McNish S, Banerjee AN, Couch KS, et al. Postoperative wound dehiscence: predictors and associations. Wound Repair Regen. 2015;23(2):184-90. http://doi. org/10.1111/wrr.12268

4. Ramshorst GH, Nieuwenhuizen J, Hop WC, Arends P, Boom J, Jeekel $\mathrm{J}$, et al. Abdominal wound dehiscence in adults: development and validation of a risk model. World J Surg. 2010;34:20-7. http://doi. org/10.1007/s00268-009-0277-y

5. Ursi ES, Galvão CM. Prevenção de lesões de pele no perioperatório: revisão integrativa da literatura. Rev. Latino-am Enferm. 2006;14(1):124-31. http://doi.org/10.1590/S0104-11692006000100017

6. Pavlidis TE, Galatianos IN, Papaziogas BT, Lazaridis CN, Atmatzidis KS, Makris JG, et al. Complete dehiscence of the abdominal wound and incriminating factors. Eur J Surg. 2001;167(5):351-4. https:// doi.org/10.1080/110241501750215221

7. Sandy-Hodgetts K, Leslie GD, Parsons R, Zeps N, Carville K. Prevention of postsurgical wound dehiscence after abdominal surgery with NPWT: a multicentre randomized controlled trial protocol. J Wound Care. 2017;26(Supl. 2):S23-6. http://doi.org/10.12968/jowc.2017.26.Sup2.S23

8. Vuolo JC. Assessment and management of surgical wounds in clinical practice. Nurs Stand. 2006;20(52):46-56. http://doi.org/10.7748/ ns2006.09.20.52.46.c4494

9. Walming S, Angenete E, Block M, Bock D, Gessler B, Haglind E. Retrospective review of risk factors for surgical wound dehiscence and incisional hernia. BMC Surg. 2017;17:19. http://doi.org/10.1186/ s12893-017-0207-0

10. Mir MA, Manzoor F, Singh B, Sofi IA, Rameez AZ, Farooq SI. Development of a risk model for abdominal wound dehiscence. Surgical Sci. 2016;7(10):466-74. http://doi.org/10.4236/ss.2016.710063

11. Spiliotis J, Tsiveriotis K, Datsis AD, Vaxevanidou A, Zacharis G, Giafis $\mathrm{K}$, et al. Wound dehiscence: is still a problem in the 21 th century: a retrospective study. World J Emerg Surg. 2009;4:12. http://doi. org/10.1186/1749-7922-4-12

12. Yılmaz KB, Akıncı M, Doğan L, Karaman N, Özaslan C, Atalay C. A prospective evaluation of the risk factors for development of wound dehiscence and incisional hernia. Ulus Cerrahi Derg. 2013;29(1):25-30. http://doi.org/10.5152/UCD.2013.06

13. Butler LD. Early postoperative complications following ostomy surgery: a review. J Wound Ostomy Continence Nurs. 2009;36(5):513-9. http:// doi.org/10.1097/WON.0b013e3181b35eaa
14. Souza MT, Silva MD, Carvalho R. Revisão integrativa: o que é e como fazer. Einstein. 2010;8(1):102-6. http://doi.org/10.1590/ s1679-45082010rw1134

15. Aromataris E, Munn Z, editors. Joanna Briggs Institute Reviewer's Manual [Internet]. The Joanna Briggs Institute; 2017 [acessado em 15 mar. 2019]. Disponível em: https://reviewersmanual. joannabriggs.org/

16. Scalise A, Calamita R, Tartaglione C, Pierangeli M, Bolletta E, Gioacchini M, et al. Improving wound healing and preventing surgical site complications of closed surgical incisions: a possible role of Incisional Negative Pressure Wound Therapy: a systematic review of the literature. Int Wound J. 2016;13(6):1260-81 . http://doi.org/10.1111/ iwj.12492

17. Sandy-Hodgetts K, Watts R. Effectiveness of negative pressure wound therapy/closed incision management in the prevention of post-surgical wound complications: a systematic review and meta-analysis. JBI Database System Rev Implement Rep. 2015;13(1):253-303. http:// doi.org/10.11124/jbisrir-2015-1687

18. Petito EL, Esteves MT, Elias S, Facina G, Nazário AC, Gutiérrez MG. The influence of the initiation of an exercise programme on seroma formation and dehiscence following breast cancer surgery. J Clin Nurs. 2014;23(21-22):3087-94. http://doi.org/10.1111/jocn.12544

19. Blume PA, Key JJ, Thakor P, Thakor S, Sumpio B. Retrospective evaluation of clinical outcomes in subjects with split-thickness skin graft: comparing VAC ${ }^{\circ}$ therapy and conventional therapy in foot and ankle reconstructive surgeries. Int Wound J. 2010;7(6):480-7. http:// doi.org/https://doi.org/10.1111/j.1742-481X.2010.00728.x

20. World Union of Wound Healing Societies (WUWHS). Consensus Document. Surgical wound dehiscence: Improving prevention and outcomes [Internet]. 2018 [acessado em 15 mar. 2019]. Disponível em: www.woundsinternational.com

21. Paiva CSB, Oliveira SMJV, Francisco AA, Silva RL, Mendes EPB, Steen M. Length of perineal pain relief after ice pack application: a quasi-experimental study. Women Birth. 2016;29(2):117-22. http:// doi.org/10.1016/j.wombi.2015.09.002

22. Kenig J, Richter P, Żurawska S, Lasek A, Zbierska K. Risk factors for wound dehiscence after laparotomy: clinical control trial. Pol Przegl Chir. 2012;84(11):565-73. https://doi.org/10.2478/v10035-012-0094-0

23. Aksamija G, Mulabdic A, Rasic I, Aksamija L. Evaluation of risk factors of surgical wound dehiscence in adults after laparotomy. Med Arch. 2016;70(5):369-72. http://doi.org/10.5455/medarh.2016.70.369-372

24. Gomes E, Marinho M, Galvão M, Rego D, Vieira J, Santos ML. Lesão por abrasão após cateterismo cardíaco: relato de caso. Rev SOBECC. 2018;23(2):109-13. http://doi.org/10.5327/Z1414-4425201800020009

25. World Health Organization. Global guidelines for the prevention of surgical site infection [Internet]. World Health Organization; 2016 [acessado em 15 mar. 2019]. Disponível em: http://www.who.int/ gpsc/ssi-prevention-guidelines/en/ 